\title{
Anti-inflammatory effect of unripe apple polyphenols-chitooligosaccharides microcapsule against LPS-induced RAW 264.7 cells
}

\author{
Hu-Zhe Zheng ${ }^{1,2}$,, Chun-Lan Cui ${ }^{1}$, Woo-Sik Jeong ${ }^{2}$ and Shin-Kyo Chung ${ }^{2^{*}}$
}

\begin{abstract}
In order to improve the synergistic effect of unripe apple polyphenols (APP) and chitooligosaccharides (COS), apple polyphenols-chitooligosaccharides microcapsule (APCM) were prepared by spray-drying method. The effects of APCM on the release of polyphenols in simulated gastrointestinal digestion model, as well as the anti-inflammatory effect against LPS-induced RAW264.7 cells were also evaluated. Scanning electron microscope (SEM) and HPLC analysis of APP and APCM showed that during the spray-drying process, most of the polyphenols are successfully encapsulated in COS. The simulated gastrointestinal digestion model results showed that about $98 \%$ of polyphenols released from APCM within 60 min. Anti-inflammatory effect of APCM on LPS induced RAW 264.7 cells showed that although APP showed a strong inhibitory effect on cell viability at $0.6 \mathrm{mg} / \mathrm{mL}$, the effect of APCM on cell viability was less and could maintain a high level at the same concentration. In addition, APCM significantly inhibited nitric oxide (NO) and TNF-a production via the elevation of cytokine IL-10 as the concentration increases, respectively. The results suggest that APCM alleviate the intensity of inflammatory processes by inhibiting the production of pro-inflammatory cytokines TNF-a, as well as additionally by promoting the production of anti-inflammatory cytokines IL-10. These findings provide scientific and theoretical support for the claim that traditional medicine treats inflammation-related diseases.
\end{abstract}

Keywords: Apple polyphenols-chitooligosaccharides microcapsule, Simulated gastrointestinal digestion model, Cell viability, Nitric oxide, TNF-a, IL-10, RAW 264.7 cells

\section{Introduction}

Apples and their products are widely recognized as important components of the human health food menu, because they contain a variety of nutrients and health benefit constituents, such as organic acids, dietary fiber, vitamins and polyphenols. Among them, polyphenols have been reported to have a variety of biological activities, such as anti-allergic activity [1], anti-arteriosclerosis activity [2] and anti-inflammatory activity [3]. Unripe

\footnotetext{
*Correspondence: 2547758626@qq.com; kchung@knu.ac.kr

${ }^{2}$ School of Food Science \& Biotechnology, Kyungpook National University, Daegu 41566, Republic of Korea

Full list of author information is available at the end of the article
}

apples, are defined as fallen and thinned-out apples in orchards, which have been reported to contain abundant amounts of polyphenols. Although the rate of fallen and thinned-out apples as high as $30 \%$ of total apple production, only a small part of them were used as raw material for animal feed or fertilizer [4].

In recent years, agricultural wastes from plant sources have received increasing attention as potential sources of pharmaceutical and food raw materials. However, in many cases research on the use of biological waste for the development of new products is not carried out systematically, and therefore extraction and deep processing 
techniques have been often regarded as bottlenecks in the food R \& D [5].

Recently, in order to improve the extraction efficiency of polyphenols in plants, carbohydrate hydrolases are often introduced to release complex polyphenols from cell walls. For instance, used for extraction of polyphenols from apple pomace [6] and unripe apple [4]. In addition, the production of apple polyphenols (APP) powder was also developed by adsorption-desorption process using Amberlite XAD series resins [7].

Chitooligosaccharides (COS), which is obtained through the decomposition of chitosan, can be dissolved in water easily to give versatile bioactive functions [8]. In addition, COS have many excellent functional properties that are not possessable in native chitosan, such as advancing anti-microbial [9] and anti-inflammatory activity [10], improving delivery of drug lesion targeting sites [11], enhancing human absorption rate by synthesis of nano-carriers with functional compounds [12]. However, COS has a little limitation, such as narrow range of application, and less powerful [13], etc. To overcome this drawbacks, COS was modificated with functional groups obtained derivatives, such as COS-Nisin conjugate [9], cinnamol-COS polymer [13], chlorogenic acid-COS conjugates [14], tea catechins-COS conjugation [15], salicylic acid-grafted COS [16], etc. The health benefits functions through mixing various functional groups with $\mathrm{COS}$ were improved significantly.

The objectives of this study were development of the unripe apple polyphenols-chitooligosaccharides microcapsule (APCM), evaluation of the effect of simulated gastrointestinal digestion model on polyphenols release and anti-inflammatory activities, such as cell viability, nitric oxide (NO), TNF- $\alpha$ and IL-10 have been executed and studied. This study will provide a clue to elucidate the APCM, which would contribute to development in the field of functional food or drugs against inflammatory diseases.

\section{Materials and methods Sample material}

Unripe apples (Malus pumila cv. Fuji) were collected at the 85 days after full bloom from the orchard of Kyungpook National University in Daegu, Korea in 2016, and stored in a freezer $\left(-70^{\circ} \mathrm{C}\right)$ until the experiment.

\section{Reagents and instruments}

Viscozyme L (from Aspergillus aculeatus, 100 fungal $\beta$-glucanase units (FBG)/mL) purchased from Novozymes (Bagsvaerd, Denmark). COS (MV, $\left.3.4 \times 10^{4} \mathrm{Da}\right)$ were purchased from KITTO LIFE Co. (Pyongtaeg, Korea). Folin Ciocalteu reagent, caffeic acid, $p$-coumaric acid, quercetin-3-glucoside, phloridzin, phloretin, 3-(4,5 dimethylthiazol-2-yl)-2,5-diphenyltetrazolium bromide (MTT), fetal bovine serum (FBS) and Dulbecco's modified Eagle's medium (DMEM) were obtained from Sigma Co. (St. Louis, MO, USA). XAD-7 resins were purchased from Rohm \& Haas Co. (Chauny, France). The RAW 264.7 cells were purchased from the American Type Culture Collection (ATCC). The other reagents were obtained from Duksan Co. (Seoul, Korea). Mini spraydryer used Büchi B-191 (Flavil, Switzerland), scanning electron microscope (SEM) was used S-570 (Hitachi, Japan), HPLC/UVD was used SPD-10A (Shimadzu, Japan), and Multilabel counter was used Victor 31420 (PerkinElmer, USA).

\section{Preparation of apple polyphenols}

Ten kilograms of whole unripe apples were blanched at $85^{\circ} \mathrm{C}$ for $15 \mathrm{~min}$ for the inhibition of polyphenol oxidase, crushed, and homogenized with equal volume of water. After addition of $1.95 \%$ Viscozyme L, the homogenized solution was incubated at $47.1{ }^{\circ} \mathrm{C}$, for $12.5 \mathrm{~h}$, filtered, and concentrated to apple crude polyphenols with a rotary evaporator. And then the sample was applied onto Amberlite XAD-7 column $(100 \mathrm{~cm} \times 7 \mathrm{~cm})$, washed out with deionized water, and eluted with $95 \%$ ethanol. The eluted solution was collected, concentrated, and finally APP powder was achieved by spray-drying process with the same method of preparation APCM [4]. As a control, without Viscozyme L treatment was used with the other same method of preparation apple polyphenols.

\section{Preparation of APCM}

According to our previous study, APP and COS powders were dissolved in 20 times distilled water, respectively, and mixed at a ratio of 2.5:1 (w/w), thoroughly stirred before the spray drying process. Spray-drying was done using Büchi B-191 spray dryer with the inlet temperature of $150{ }^{\circ} \mathrm{C}$, pump setting of $40 \%$, air flow rate of $125 \mathrm{~m}^{3} / \mathrm{h}$, aspirator set at $60 \%$ and outlet temperature of $65{ }^{\circ} \mathrm{C}$, the solution flow rate was $250 \mathrm{~mL} / \mathrm{h}$. As a contrast, COS was performed the same spray-drying process.

\section{SEM analysis}

COS, APP and APCM were subjected to study SEM analysis. SEM was carried out at $15 \mathrm{kV}$. The samples were sprinkled on to conductive glue on an aluminum SEM stub and sputter coated with gold.

\section{Analysis of polyphenols content and composition}

Determination of total phenolic content (TPC) was according Folin-Ciocalteu method and expressed as gallic acid equivalents ( $\mathrm{mg}$ GAE/g). The investigation of polyphenol composition was performed on an HPLC equipped with a UV-Visible detector at $290 \mathrm{~nm}$ using an 
ODS-HG-5 column (Develosil, $150 \times 4.6 \mathrm{~mm}$, i.d.) and a security guard C18 ODS $(4 \times 3.0 \mathrm{~mm}$, i.d.). The mobile phase consisted of $2 \%(\mathrm{v} / \mathrm{v})$ acetic acid in water (eluent A) and $0.5 \%(\mathrm{v} / \mathrm{v})$ acetic acid and $49.5 \%(\mathrm{v} / \mathrm{v})$ acetonitrile in water (eluent $\mathrm{B}$ ). The gradient was programmed as follows: $12-25 \% \mathrm{~B}(0-15 \mathrm{~min}), 25-35 \% \mathrm{~B}$ (15-25 min), 35-55\% B (25-50 min), 55-65\% B (50-60 min), and $65-12 \%$ B (60-70 $\mathrm{min})$. The flow rate and injection volume were $1.0 \mathrm{~mL} / \mathrm{min}$ and $20 \mu \mathrm{L}$, respectively [14].

\section{Release ratio of polyphenols from APCM in simulated gastrointestinal digestion model}

In vitro release ratio of polyphenols from APCM was detected in simulated gastric fluid (SGF) and simulated intestinal fluid (SIF) by a modification of the method of Pasukamonset et al. [17]. Briefly, the sample was treated with SGF (1.0 g of NaCl, $12 \mathrm{~mol} / \mathrm{L} \mathrm{HCl}, 3.5 \mathrm{~mL}, \mathrm{pH} 1.68)$ at $37{ }^{\circ} \mathrm{C}$ with agitation, for $0-30 \mathrm{~min}$. Then, the $\mathrm{pH}$ was adjusted to SIF $\left(0.2 \mathrm{~mol} / \mathrm{L} \mathrm{NaOH}, 0.2 \mathrm{~mol} / \mathrm{L} \mathrm{KH}_{2} \mathrm{PO}_{4}, \mathrm{pH}\right.$ 6.8), followed by incubation at $37{ }^{\circ} \mathrm{C}$ with agitation for 0-30 min. APP as a control was performed. The samples as a pair, were simultaneously divided into 6 parts, each of which was prepared for each subsequent experiment, respectively. And then, the solution was used to determine TPC and polyphenols content and composition.

\section{Cell viability}

For cell viability assays, RAW 264.7 cells were seeded into a 96-well plate with a density of $5 \times 10^{5}$ cells/wells and incubated with the medium in the presence of sample at different concentrations. After incubation for $24 \mathrm{~h}$, media change to FBS free DMEM $100 \mu \mathrm{L}, 5 \mathrm{mg} / \mathrm{mL}$ MTT $10 \mu \mathrm{L}$ was added and continuous incubated for another $1 \mathrm{~h}$. Victor 31420 Multilabel counter was then used to dissolve the nitrogen crystal in dimethyl sulfoxide and measure the absorbance at $595 \mathrm{~nm}$ [18].

\section{Nitric oxide production}

Nitric oxide (NO) production was detected according Griess reaction method [3]. First, RAW 264.7 cells with a density of $5 \times 10^{5}$ cells/wells were plated in 96 -well plates, incubated for $24 \mathrm{~h}$ and pretreated with the sample at different concentrations for $1 \mathrm{~h}$, and then challenged with LPS $(5 \mu \mathrm{g} / \mathrm{mL})$ for an additional $24 \mathrm{~h}$. After incubation, mixed with the same volume of Griess reagent and incubated for $10 \mathrm{~min}$. After then, the mixture was measured at $540 \mathrm{~nm}$ with a Victor 31420 Multilabel counter, and the absorption coefficient was calibrated using a sodium nitrite solution standard.

\section{TNF-a production}

TNF- $\alpha$ production induced in RAW 264.7 cells was performed by the method of Pantea et al. [19]. Briefly, cells with a density of $1 \times 10^{5}$ cells/well in 96-well plates were treated with $10 \mu \mathrm{L}$ of different concentrations of sample, and the treated with $1 \mu \mathrm{g} / \mathrm{mL}$ of LPS and incubated for $24 \mathrm{~h}$. After then, $100 \mu \mathrm{L}$ of supernatants was moved to ELISA plate from media, and incubate for $2 \mathrm{~h}$ at $20{ }^{\circ} \mathrm{C}$. And then add $50 \mu \mathrm{L}$ of TNF- $\alpha$ antibody to each well and incubate for $2 \mathrm{~h}$ at $20^{\circ} \mathrm{C}$. Add $50 \mu \mathrm{L}$ of streptavidin-HRP to each well and incubate for $2 \mathrm{~h}$ at $20^{\circ} \mathrm{C}$. Finally, determine the optical density of each well with a Victor 31420 Multilabel counter at $450 \mathrm{~nm}$.

\section{IL-10 production}

IL-10 production in RAW 264.7 cells was detected using ELISA method following the manufacturer's protocol [19], which is similar to TNF- $\alpha$ assay method.

\section{Statistical analysis}

Data were presented as means \pm standard deviations (S.D.). Values were evaluated by analysis of variance (ANOVA). $p<0.05$ was considered as significantly difference.

\section{Results and discussions}

Polyphenols content and composition of APP and APCM

Polyphenols with various physiological activities are mainly present in fruits and seeds. However, many polyphenols remain in apple pomace during processing [2]. Hence, carbohydrate hydrolyzing enzymes, Viscozyme L assisted method was used to improve the extraction effect of unripe apple polyphenols in this study. After enzyme-aided extraction, XAD-7 adsorption was used the process. The extraction as well as the content and composition results are shown in Table 1. The results showed that compared with the control group, the extraction TPC rate in Viscozyme L aided treatment

Table 1 Phenolics content of APP and APCM

\begin{tabular}{|c|c|c|c|c|c|c|c|}
\hline \multirow[t]{2}{*}{ Product } & \multirow[t]{2}{*}{ TPC (mg GAE/g) } & \multicolumn{6}{|c|}{ Polyphenols composition (mg/g) } \\
\hline & & Chlorogenic acid & Caffeic acid & $p$-Coumaric acid & Quercetin-3-glucoside & Phloridzin & Phloretin \\
\hline Control & $247.42 \pm 1.63$ & $108.10 \pm 0.87$ & $2.69 \pm 0.13$ & $0.91 \pm 0.09$ & $0.29 \pm 0.01$ & $17.48 \pm 0.89$ & $0.43 \pm 0.11$ \\
\hline APP & $498.72 \pm 2.52$ & $7.29 \pm 0.59$ & $36.16 \pm 0.37$ & $10.22 \pm 0.21$ & $13.31 \pm 0.33$ & $8.73 \pm 0.22$ & $3.16 \pm 0.23$ \\
\hline APCM & $355.27 \pm 1.29$ & $5.13 \pm 0.16$ & $25.33 \pm 0.42$ & $7.36 \pm 0.68$ & $9.58 \pm 0.41$ & $6.37 \pm 0.17$ & $2.31 \pm 0.18$ \\
\hline
\end{tabular}


increased by about 2 times, indicating that the hydrolysis reaction was more active under enzyme-aided conditions. In addition, as the main phenolic compounds in apple fruits, chlorogenic acid, caffeic acid, $p$-coumaric acid, quercetin-3-glucoside, phloridzin, and phloretin were detected with and without Viscozyme L aided treatment, respectively. Table 1 shown chlorogenic acid $(108.10 \mathrm{mg} / \mathrm{g})$ content has the highest, followed by phloridzin $(17.48 \mathrm{mg} / \mathrm{g})$, caffeic acid $(2.69 \mathrm{mg} / \mathrm{g}), p$-coumaric acid $(0.91 \mathrm{mg} / \mathrm{g})$, phloretin $(0.43 \mathrm{mg} / \mathrm{g})$ and quercetin3 -glucoside $(0.29 \mathrm{mg} / \mathrm{g})$ in without Viscozyme L treated control group, respectively. However, the polyphenols composition of APP was significantly different compared with control, which reflects the decrease of chlorogenic acid (14.8 times) content associated with the increase of caffeic acid (13.4 times) content (Table 1). In addition, compared with the control, $p$-coumaric acid and quercetin-3-glucoside content increased about 11.2 and 45.9 times, respectively. According to previous research indicated that caffeic acid and $p$-coumaric acid were widely regarded as important indicators of the decomposition of plant cell walls during enzymatic hydrolysis [7], which could be explain the result of increase of caffeic acid and $p$-coumaric acid by the carbohydrate-hydrolyzing enzyme Viscozyme L (Table 1). Genaro-mattos et al. [20] announced caffeic acid has excellent anti-inflammatory, anti-mutagenic and anti-cancer effects. In addition, Caroline et al. [21] even investigated caffeic acid acting as an inflammatory inhibitor. Thus, an even more pronounced anti-inflammatory activity of APCM can be guaranteed, allowing for further studies. The APCM was obtained by the mixture ratio of APP and COS 2.5:1 (w/w) before the spray-drying process. Table 1 alos showed polyphenols content and composition of APCM. As expected, the content and composition of APCM exhibited about 70\% level of APP, which means during the spray-drying process, most of the polyphenols are successfully encapsulated in COS, to minimize the loss of polyphenols during processing.

\section{Morphology of APCM}

As a drugs and nutrients carrier, COS helps overcome certain adverse characteristics of drugs and nutrients, such as insolubility and hydrophobicity [22]. However, the disadvantage of the narrow scope of COS limits its application as active functional food [13]. In recent years, the interactions between $\operatorname{COS}$ and green tea extract [1], strawberry extract [23], and apple peel extract [24], have been studied respectively. These days, spray-drying technique has been developed for drug making associated with COS [25]. Since, drug release properties may be generated through the inclusion of drug release modifiers during spray-dried process [26]. In this study, in order to improve bioavailability and delivery of apple polyphenols to target cells by drug carrier COS, APCM was produced by spray-drying technique. Figure 1 shows the SEM of the APCM, as well as COS and APP. As can be seen, the COS appeared to have many wrinkles on the surface and there were gaps between the wrinkles (Fig. 1a). However, APCM had a smooth and spherical surface morphology except for pores in larger microspheres observed at higher magnification, with diameters between $20 \mu \mathrm{m}$ and $50 \mu \mathrm{m}$. Additionally, it was found in particle size analysis that APCM have smaller particles and better redispersibility than APP or COS (data not shown). The similar observation was recorded by Bruna et al. [26], who studied chitosan-encapsulated Plinia cauliflora extract, and confirmed that smaller particles had a larger surface area resulting in a faster degradation. In addition, according to our previous research inferred that the vast majority of APCM was obtained by physical combining, due to hydrogen bonding, electrostatic and van der Waals interactions between COS and APP [27]. Thus, APCM were able to maintain APP and COS their respective excellent health functions. So, it was expected that APCM can better stabilize health benefit functions compounds of the release than COS and APP.
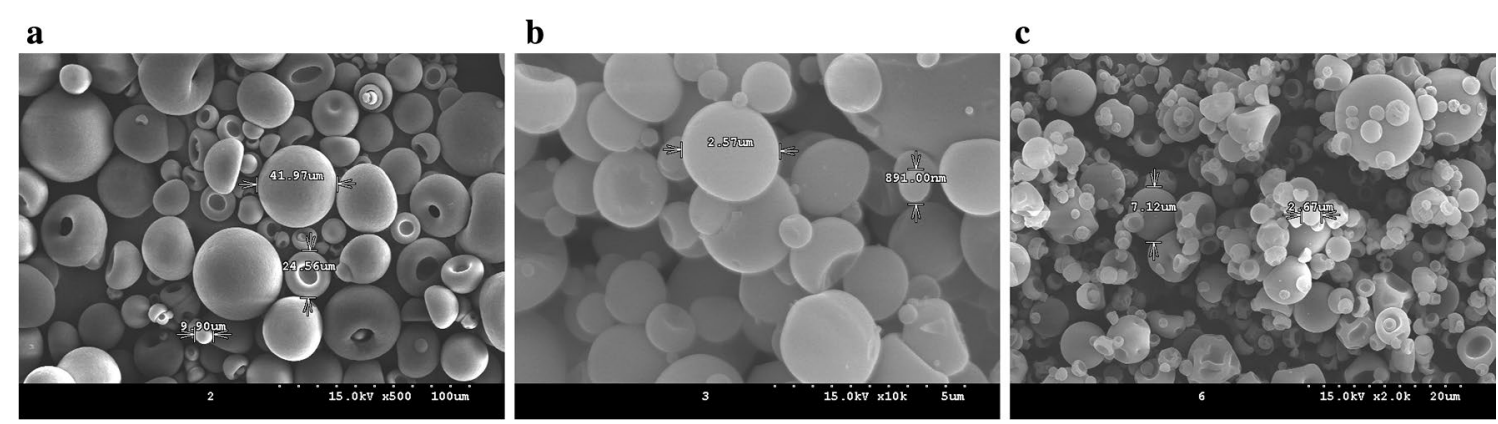

Fig. 1 SEM photographs of APCM obtained by spray-drying using COS as an encapsulating agent. a COS, b APP, c APCM 
Release ratio of polyphenols from APCM in simulated gastrointestinal digestion model

To further understand the polyphenols absorption on human body, the study of polyphenols release effect from APCM was necessary. The polyphenols relealesed from APP and COS encapsulated APCM in SGF (pH 1.68) and SIF ( $\mathrm{pH}$ 6.8) digestion model were demonstrated in Fig. 2 and Table 2. The results showed that the release of polyphenols from APP and APCM occurred within $60 \mathrm{~min}$. As a control treatment, APP showed excellent TPC and polyphenols release capacity. In addition, TPC released from APCM ranged from $55.17 \%$ to $98.45 \%$ during the incubation periods, which similar to APP release ratio of $57.23 \%$ to $99.87 \%$. In addition, the release ratio of polyphenols from APCM showed similar pattern compared with APP. Recently, many researches indicate that after release in stomach and small-intestine, unreleased polyphenols could be further released in large-intestine for prolonged periods [17]. Since, further studies on the release and absorb by human body are required.

\section{Anti-inflammatory effect of APCM against LPS-induced RAW 264.7 cells}

As an endotoxin, LPS is the principal component of the outer membrane of gram-negative bacteria, which could induce production of inflammatory mediators such as NO, TNF- $\alpha$, IL-1, IL-8 [28], etc. Therefore, LPS induced RAW264.7 cells have been used to evaluate the anti-inflammatory effects of various materials. Moreover, it was agreed that LPS induces pro-inflammatory cytokine IL-10 production, which can down-regulates inflammatory response such as NO, TNF- $\alpha$ and IL-1 [3], etc. Recently research also corroborated that inflammation is an important chronic trigger [29]. Fortunately,
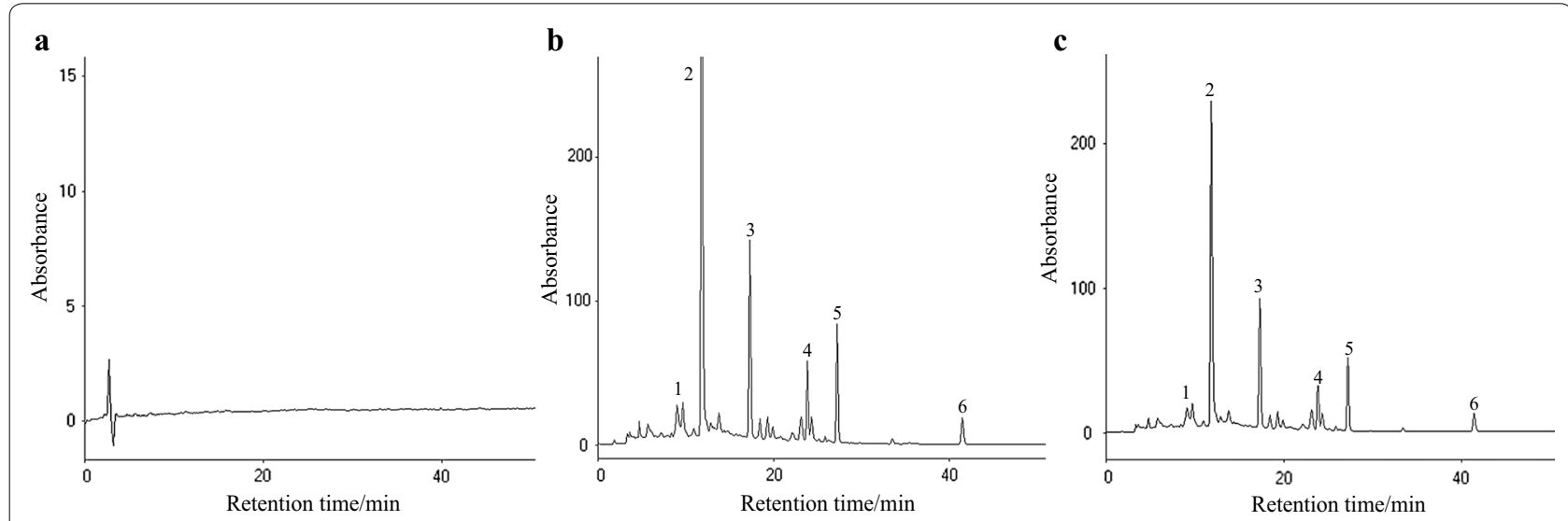

Fig. 2 HPLC chromatograms of polyphenols released from APP and COS encapsulated APCM in simulated gastrointestinal digestion model after 60 min. A COS; B APP; C APCM. 1 chlorogenic acid, 2 caffeic acid, 3 p-coumaric acid, 4 quercetin-3-glucoside, 5 phloridzin, 6 phloretin

Table 2 Polyphenols release rates from APP and APCM in simulated gastrointestinal digestion model

\begin{tabular}{|c|c|c|c|c|c|c|}
\hline \multirow[t]{3}{*}{ Polyphenols } & \multicolumn{6}{|c|}{ Polyphenols content (release rate, \%) } \\
\hline & \multicolumn{2}{|l|}{$20 \mathrm{~min}$} & \multicolumn{2}{|l|}{$40 \mathrm{~min}$} & \multicolumn{2}{|l|}{$60 \mathrm{~min}$} \\
\hline & APP & APCM & APP & APCM & APP & APCM \\
\hline TPC (mg GAE/g) & $\begin{array}{l}285.05 \pm 2.21 \\
(57.23)^{*}\end{array}$ & $196.01 \pm 1.71(55.17)$ & $\begin{array}{l}398.01 \pm 1.71 \\
(79.91)^{*}\end{array}$ & $260.05 \pm 1.95(73.31)$ & $498.07 \pm 2.13(99.87)$ & $349.76 \pm 1.27(98.45)$ \\
\hline $\begin{array}{l}\text { Chlorogenic acid } \\
(\mathrm{mg} / \mathrm{g})\end{array}$ & $4.24 \pm 0.21(58.21)^{*}$ & $2.89 \pm 0.14(56.43)$ & $5.84 \pm 0.29(80.05)^{*}$ & $3.80 \pm 0.42(74.12)$ & $7.28 \pm 0.27$ (99.81) & $5.04 \pm 0.12(98.42)$ \\
\hline Caffeic acid (mg/g) & $21.02 \pm 0.83(58.13)^{*}$ & $14.27 \pm 0.53(56.34)$ & $28.77 \pm 0.43(79.57)^{*}$ & $18.81 \pm 0.55$ & $36.01 \pm 0.53(99.57)$ & $24.88 \pm 0.43(98.24)$ \\
\hline $\begin{array}{l}p \text {-Coumaric acid } \\
(\mathrm{mg} / \mathrm{g})\end{array}$ & $5.81 \pm 0.84(56.87)$ & $4.08 \pm 0.62(55.46)$ & $8.05 \pm 0.34(78.75)^{*}$ & $5.43 \pm 0.48$ & $10.15 \pm 0.45(99.28)^{*}$ & $7.20 \pm 0.48(97.89)$ \\
\hline $\begin{array}{l}\text { Quercetin-3-gluco- } \\
\text { side }(\mathrm{mg} / \mathrm{g})\end{array}$ & $7.66 \pm 0.63(57.57)^{*}$ & $5.21 \pm 0.43(54.39)$ & $10.56 \pm 0.36(79.34)^{*}$ & $6.97 \pm 0.42(72.79)$ & $13.24 \pm 0.47$ (99.49) & $9.41 \pm 0.37(98.21)$ \\
\hline Phloridzin (mg/g) & $5.02 \pm 0.42(57.42)^{*}$ & $3.47 \pm 0.32(54.44)$ & $6.84 \pm 0.31(78.34)^{*}$ & $4.70 \pm 0.28(73.74)$ & $8.71 \pm 0.34(99.81)^{*}$ & $6.24 \pm 0.33(97.94)$ \\
\hline Phloretin (mg/g) & $1.80 \pm 0.21(56.88)$ & $1.29 \pm 0.21(55.83)$ & $2.52 \pm 0.26(79.76)^{*}$ & $1.68 \pm 0.31(72.71)$ & $3.14 \pm 0.36(99.29)$ & $2.28 \pm 0.37(98.78)$ \\
\hline
\end{tabular}

* APCM group compared with APP group $(p<0.05)$ 
developing scientific evidence suggests that many antioxidant compounds such as polyphenols-rich fruits, vegetables and other plants have a strong potential to regulate biological pathways to prevent or reduce inflammatory diseases [19]. In this study, anti-inflammatory effect of APCM against LPS-induced RAW 264.7 cells were investigated. APP and COS as control were performed. RAW 264.7 cells were treated with the concentration of 0.1 , 0.3 and $0.6 \mathrm{mg} / \mathrm{mL}$, respectively, and then induced NO, TNF- $\alpha$ and IL-10 productions were determined to evaluate the anti-inflammatory effect.

\section{Cell viability}

In order to determine the effect of APP on cell toxicity, different concentrations of products were used to test RAW 264.7 cytotoxicity to establish a suitable APCM concentration range. The results indicated that as increase APP concentration, cell viability decreased rapidly, and decreased to half when concentration reached to $0.6 \mathrm{mg} / \mathrm{mL}$ (Fig. 3). This result suggest that high concentrations of APP produce high toxicity. This result was supported by Du et al. [30], who reported that cytotoxic effect was less significant in low concentrations of peel fruit extract, however significantly affected by relative high concentration of polyphenols [31]. In case of COS, cell viability was not affected by concentrations and could be maintained high level at different concentrations. On the other hand, APCM displayed a similar inhibitory effect with COS. These results indicate that APCM enhances the cell viability, which was probably due to the synergy effect of COS and APP. This result was supported by Rui et al. [14], who pronounced that chlorogenic acid-COS conjugates significantly increased cell viability

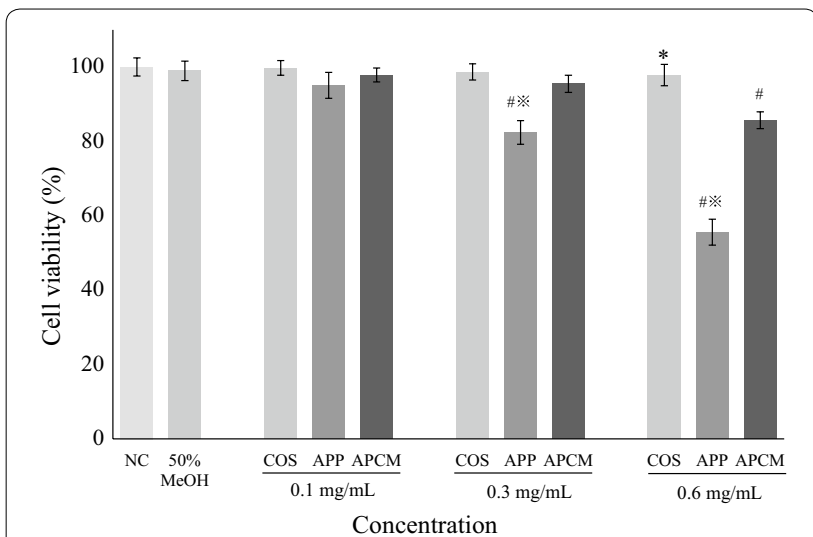

Fig. 3 Effects of COS, APP and APCM on cell viability against LPS-induced RAW 264.7 cells. Results were expressed as mean \pm S.D.

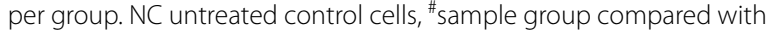
NC $(p<0.05),{ }^{*} A P C M$ group compared with COS group $(p<0.05)$, *APCM group compared with APP group $(p<0.05)$ compared with chlorogenic acid. According to the above results, the cytotoxicity is mainly caused by polyphenols themselves, COS microcapsules have little effect in apoptosis. In addition, according to Liang et al. [32], who reported that COS-coated tea polyphenols nanoparticles increased cell viability, which was in a dose-dependent manner.

\section{NO production}

The anti-inflammatory effects of APP on the NO production in LPS-induced RAW 264.7 cells were evaluated. As shown in Fig. 4, during incubation time of $24 \mathrm{~h}$, RAW 264.7 cells produced $3.51 \mu \mathrm{M}$ NO in the untreated control cells. The results indicated that LPS stimulated RAW 264.7 cells promoted production of NO to $28.73 \mu \mathrm{M}$. In the case of the APP treatment showed the highest NO produced inhibition in a dose dependent manner corresponding to $41.83 \%(14.71 \mu \mathrm{M}), 52.17 \%(13.74 \mu \mathrm{M})$ and $68.78 \%(8.97 \mu \mathrm{M})$ inhibition at the concentration of $0.1,0.3$ and $0.6 \mathrm{mg} / \mathrm{mL}$, respectively, compared with untreated control cells (NC) and APCM. Our results were supported by Leyva et al. [3], who resulted that NO production was significantly inhibited by oregano leaves extract due to high levels of polyphenols. In our case, APP contained rich polyphenols, about $498.72 \mathrm{mg}$ GAE/g (Table 1), and this may explain why APP have a more significant effect on decreasing NO accumulation. Furthermore, according to Rui et al. [14] and Gema et al. [18], NO may bind to oxygen free radicals to form potent oxidants involved in oxidative damage to cells and tissues. Therefore, by enhancing ROS activity, to inhibit NO production $[4,28]$. Notably, in agreement with previous studies of antioxidant and ROS scavenging activities

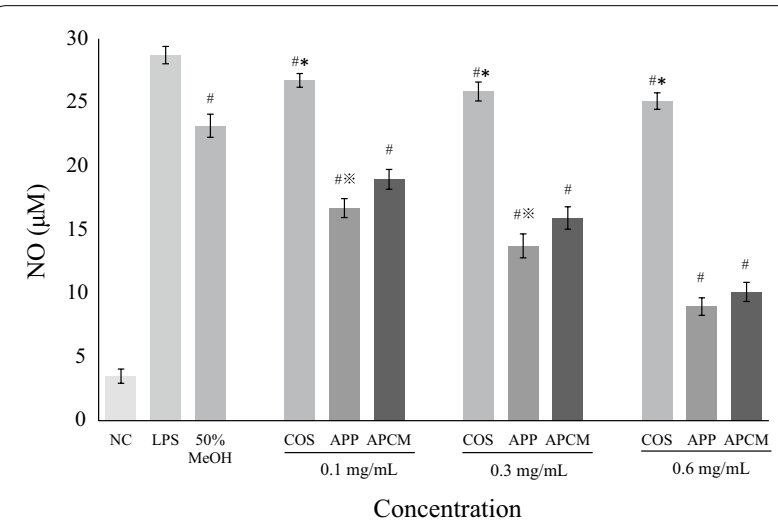

Fig. 4 Effects of COS, APP and APCM on NO production against LPS-induced RAW 264.7 cells. Results were expressed as mean \pm S.D. per group. NC untreated control cells, LPS only LPS $(5 \mu \mathrm{g} / \mathrm{mL})$ treatment, \# sample group compared with LPS $(p<0.05)$, *APCM group compared with COS group $(p<0.05),{ }^{*} \mathrm{APCM}$ group compared with APP group $(p<0.05)$ 
(data not shown), APP exhibited excellent antioxidant and ROS scavenging activities, which indicated that the decrease of NO level was closely related high levels of polyphenols and its radical scavenging activities.

In case of COS, at the concentration of $0.1,0.3$, and $0.6 \mathrm{mg} / \mathrm{mL}$ showed $6.92 \%(26.74 \mu \mathrm{M}), 9.95 \%(25.12 \mu \mathrm{M})$ and $12.56 \%(20.37 \mu \mathrm{M})$ inhibition against NO production, respectively, whereas APCM showed 33.97\% (18.97 $\mu \mathrm{M})$, 45.55\% (15.93 $\mu \mathrm{M})$ and $64.78 \%(10.12 \mu \mathrm{M})$, respectively. These results indicate that APCM have better inhibitory effects of NO production than COS. In addition, with the increase of APCM concentration, especially when reaches to $0.6 \mathrm{mg} / \mathrm{mL}$, the $\mathrm{NO}$ inhibition effect was close to the same concentration APP level. Our results were supported by Eom et al. [33], who reported that COS showed relatively lower weak NO inhibition activity $(7.5 \%$ at $0.4 \mathrm{mg} / \mathrm{mL})$ whereas caffeic acid-COS conjugate $(88.3 \%$ at $0.4 \mathrm{mg} / \mathrm{mL})$ exhibited relatively higher than COS. According to above mentioned results, we can infer that NO inhibitory activity of APCM is related to its structure, due to the substitutions on the aromatic ring and the structure of the side chain COS perform as chain breaking NO production effect [27].

\section{TNF-a production}

Several studies have suggested that whether products have anti-inflammatory effects, TNF- $\alpha$ production play a key mediators [19]. The effect of TNF- $\alpha$ production regulated by COS, APP and APCM were shown in Fig. 5, very low levels of TNF- $\alpha$ are produced in RAW 264.7 cells without LPS induction. But, under the LPS induction, cells secrete high levels of TNF- $\alpha$. However, pretreatment with APP significantly reduced the production of TNF- $\alpha$ in LPS-induced RAW 264.7 cells at the concentration of 0.3 to $0.6 \mathrm{mg} / \mathrm{mL}$ from $36.55 \%$ to $65.74 \%$. Genaro-mattos et al. [20] and Zhang et al. [34] obtained similar results, who investigated that TNF- $\alpha$ levels dramatic decreased in hawthorn polyphenols and green tea polyphenols treatment, respectively compared to untreated control. In case of COS, the inhibition of TNF- $\alpha$ production was not significantly affected by its concentrations. However, the production of TNF- $\alpha$ was significantly reduced when APCM was treated in LPS-stimulated RAW 264.7 cells. At the concentration of $0.6 \mathrm{mg} / \mathrm{mL}$, APCM reduced TNF- $\alpha$ production about $61.1 \%$, which the inhibition of TNF- $\alpha$ activity of APCM was approximately 2 times higher than COS. Ngo et al. [8] suggested COS could act as immune-stimulating mediator, which could effects the levels of many inflammation factors such as TNF- $\alpha$ via the JNK/NF-kB pathways. In addition, Vo et al. [35] announced that polyphenols had anti-inflammatory properties by regulate of pro-inflammatory mediators, such as inhibition of iNOS expression and further

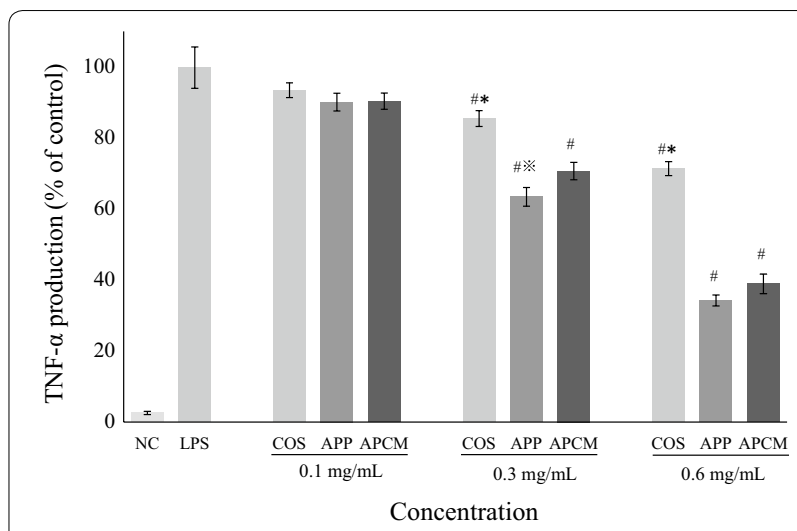

Fig. 5 Effects of COS, APP and APCM on TNF-a production against LPS-induced RAW 264.7 cells. Results were expressed as mean \pm S.D. per group. NC untreated control cells, LPS only LPS $(5 \mu \mathrm{g} / \mathrm{mL})$ treatment, \# sample group compared with LPS $(p<0.05),{ }^{*} \mathrm{APCM}$ group compared with COS group $(p<0.05)$, *APCM group compared with APP group $(p<0.05)$

NO production, TNF- $\alpha$ mRNA gene expression and TNF- $\alpha$ production. Accordingly, it was suggested that APP enhances the anti-inflammatory activities of COS as well as drug carriers when it was microcapsulated in COS. Thus, the inhibitory activity of APCM on TNF- $\alpha$ was significantly higher than that of COS. It was in agreement with the reported by Vo et al. [35], who reported that gallate grafted-COS enhanced the anti-inflammatory properties via inhibiting COX-2 expression and TNF- $\alpha$ generations.

\section{IL-10 production}

IL-10 is a typical immunomodulatory and anti-inflammatory cytokine. Recently, several researches have shown the promote effect of IL-10 production by polyphenols such as apigenin, kaempferol and resveratrol treatment in RAW 264.7 cells [36]. According to this background, this study aimed to investigate whether the anti-inflammatory effects of APCM were attributed to upregulation of anti-inflammatory cytokine IL-10. The effect of IL-10 production regulated by COS, APP and APCM were shown in Fig. 6. To antiinflammatory cytokine, as expected, pretreatment with APP significantly promoted the production of the IL-10 in LPS-induced RAW 264.7 cells from $6.6 \%$ to $49.1 \%$ at the concentration of $0.1-0.6 \mathrm{mg} / \mathrm{mL}$. As important two key mediators, $\mathrm{NO}$ and TNF- $\alpha$ positively regulate each other in host defense and inflammation process [19]. In our case, a significant reduction of TNF- $\alpha$ and NO production associated with a significant increase of IL-10 production were observed when incubating RAW 264.7 cells with the increase of APP concentration (Figs. 4, 5, 6). As expected, COS smoothly increased 


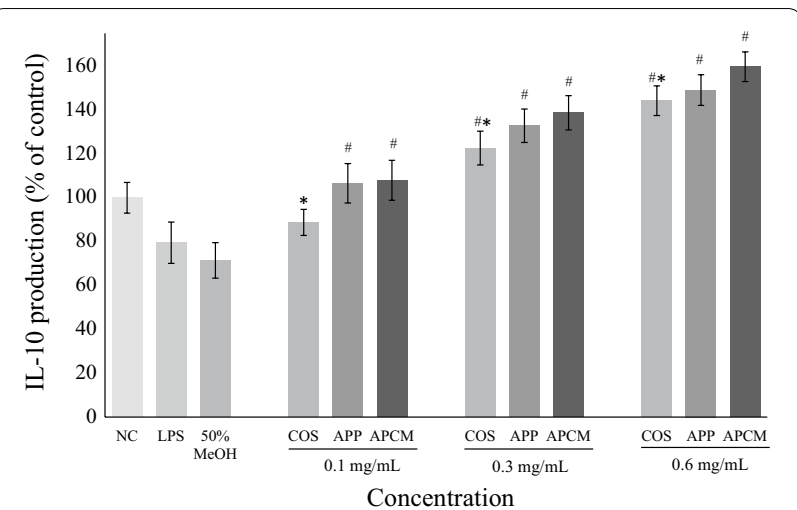

Fig. 6 Effects of COS, APP and APCM on IL-10 production against LPS-induced RAW 264.7 cells. Results were expressed as mean \pm S.D. per group. NC untreated control cells, LPS only LPS $(5 \mu \mathrm{g} / \mathrm{mL})$ treatment, \# sample group compared with LPS $(p<0.05),{ }^{*} \mathrm{APCM}$ group compared with COS group $(p<0.05)$, *APCM group compared with APP group $(p<0.05)$

IL-10 production, implying that COS can also play its anti-inflammatory role by positively regulating IL-10 expression. According to Mei et al. [37], COS may effectively improve the level of IL-10 to regulate the function of immunocompetent cells. Furthermore, IL-10 production significant increased in APCM treatment compared with the COS group, which is similar to previous observations of Nourhan et al. [38], who reported the alginate-coated chitosan nanoparticles dramatic enhanced IL-10 level compared with chitosan alone treatment, which via Th1/Th2 immune pathway.

\section{Abbreviations}

APP: Apple polyphenols; COS: Chitooligosaccharides; APCM: Apple polyphenols-chitooligosaccharides microcapsule.

\section{Acknowledgements}

This research was supported by Qinglan Project (Su Teach 2019III) in Jiangsu Province, China.

\section{Authors' contributions}

ZHZ analyzed data and wrote the manuscript and CCL analyzed polyphenols contents and composition. JWS analyzed scanning electron microscope (SEM). CSK organized this study and manuscript. All authors read and approved the final manuscript.

\section{Funding}

Funding received from Education Department of Jiangsu Province, China.

\section{Availability of data and materials}

All data analysed during this study are included in this published article.

\section{Competing interests}

The authors declare that they have no competing interests.

\section{Author details}

${ }^{1}$ Department of Health Science, Jiangsu Food \& Pharmaceutical Science College, Huai'an 223003, China. ${ }^{2}$ School of Food Science \& Biotechnology, Kyungpook National University, Daegu 41566, Republic of Korea.
Received: 1 July 2020 Accepted: 25 August 2020

Published online: 05 September 2020

\section{References}

1. Liu J, Liu YJ YJ, Pan JX (2019) Effect of tea polyphenols appended with chitosan on the shelf life of salmon. China Tea Proc 4(4):66-71

2. Barreira JCM, Ana AA, Isabel CFR (2019) Bioactive and functional compounds in apple pomace from juice and cider manufacturing: potential use in dermal formulations. Trends Food Sci Technol 90(5):76-87

3. Leyva N, Nair V, Bang WY (2016) Protective role of terpenes and polyphenols from three species of Oregano (Lippia graveolens, Lippia palmeri and Hedeoma patens) on the suppression of lipopolysaccharideinduced inflammation in RAW264.7 macrophage-cells. J Ethnopharmacol 187(7):302-312

4. Zheng HZ, Hwang IW, Kim BK, Chung SK (2014) Phenolics enrichment process from unripe apples. J Korean Soc Appl Biol 57(4):457-461

5. Perussello CA, Zhang Z, Marzocchella A (2017) Valorization of apple pomace by extraction of valuable compounds. Compr Rev Food Sci Food Saf 361(6):776-796

6. Cui CL, Zheng HZ, Gu LZ, Chung SK (2013) Optimization of pectinase aided polyphenol extraction from apple pomace by response surface methodology. Modern Food Sci Technol 29(9):2235-2240

7. Zheng HZ, Cui CL, Chung SK (2019) Identification of polyphenols from unripe apple with Viscozyme L extraction. Agric Biol 8(2):153-156

8. Ngo DH, VoTS, Ngo DN (2015) Biological effects of chitosan and its derivatives. Food Hydrocoll 51(10):200-216

9. Zheng XJ, He Y, Zhou H (2019) Preparation, characterization and antimicrobial activity of chitosan oligosaccharide-nisin conjugate. J Chinese Inst Food Sci Technol 19(11):108-115

10. Muanprasat C, Chatsudthipong V (2017) Chitosanoligosaccharide: biological activities and potential the rapeutic applications. Pharmacol Therapeut 170(2):80-97

11. Luo Y, Teng Z, Wang X (2013) Development of carboxymethyl chitosan hydrogel beads in alcohol-aqueous binary solvent for nutrientdelivery applications. Food Hydrocoll 31(2):332-339

12. Hu QB, Luo YC (2016) Polyphenol-chitosan conjugates: synthesis, characterization, and applications. Carbohydr Polym 151(20):624-639

13. Yue L, Sun D, Imran MK (2020) Cinnamyl alcohol modified chitosan oligosaccharide for enhancing antimicrobial activity. Food Chem 309(9):1-6

14. Rui LY, Xie MH, Hu B (2017) Enhanced solubility and antioxidant activity of chlorogenic acid-chitosan conjugates due to the conjugation of chitosan with chlorogenic acid. Carbohydr Polym 170(15):206-216

15. Chanphai P, Tajmir-riahi HA (2018) Conjugation of tea catechins with chitosan nanoparticles. Food Hydrocoll 84(11):561-570

16. Wang XY, Zhang L, Wei XH (2013) Molecular dynamics of paclitaxel encapsulated by salicylic acid-grafted chitosan oligosaccharide aggregates. Biomaterials 34(7):1843-1851

17. Pasukamonset P, Kwon O, Adisakwattana S (2016) Alginate-based encapsulation of polyphenols from Clitoria ternatea petal flower extract enhances stability and biological activity under simulated gastrointestinal conditions. Food Hydrocoll 61(12):772-779

18. Gema F, Concepcion P, Carmen G (2013) Methyl jasmonate treatment of strawberry fruits enhances antioxidant activity and the inhibition of nitrite production in LPS-stimulated Raw 264.7 cells. J Funct Foods 5(4):1803-1809

19. Pantea RY, Jade L, Schohraya S (2018) Apple peel polyphenols reduce mitochondrial dysfunction in mice with DSS-induced ulcerative colitis. J Nutr Biochem 57(3):56-66

20. Genaro-mattos TC, Maurício ÂQ, Rettori D (2015) Antioxidant activity of caffeic acid against Iron-induced free radical generation-a chemical. Approach. PLoS ONE 10(11):1-12

21. Caroline MS, Renata PA, Iguatemy LB (2019) In vitro methods to determine the antioxidant activity of caffeic acid. Spectrochim Acta A 219(4):358-366

22. Lee DS, Woo JY, Ahn CB (2014) Chitosan-hydroxy cinnamic acid conjugates: preparation, antioxidant and antimicrobial activity. Food Chem 148(8):97-104 
23. Rama P, Caroline M, Ratul KD (2016) Encapsulation and release studies of strawberry polyphenols inbiodegradable chitosan nanoformulation. Int Biol Macromol 88(7):171-178

24. Riaz A, Lei SC, Muhammad HS (2018) Preparation and characterization of chitosan-based antimicrobial active food packaging film incorporated with apple peel polyphenols. Int Biol Macromol 114(15):547-555

25. Dila A, Mine G, Aysun Y (2020) Stirred-type yoghurt incorporated with sour cherry extract in chitosan-coated liposomes. Food Hydrocoll 101(2):1-10

26. Bruna RPC, Paula MO, Guilherme MG (2018) Improving stability of antioxidant compounds from Plinia cauliflora (jabuticaba) fruit peel extract by encapsulation in chitosan microparticles. J Food Eng 238(11):195-201

27. Zheng HZ, Choi JH, Seong GU, Chung SK (2020) Preparation, characterization and health benefits function of unripe apple polyphenols-chitooligosaccharides microcapsule. Trans CSAE 36(14):281-289

28. Serra G, Incani A, Serreli G (2018) Olive oil polyphenols reduce oxysterolsinduced redox imbalance and proinflammatory response in intestinal cells. Redox Biol 17(7):348-354

29. Jasminka G, Damir M, Adriano P (2016) Cocoa polyphenols exhibit antioxidant, antiinflammatory, anticancerogenic, and antinecrotic activity in carbon tetrachlorideintoxicated mice. J Funct Foods 23(5):177-187

30. Du L, Li JK, Zhang XT (2018) Pomegranate peel polyphenols inhibits inflammation in LPS-induced RAW264.7 macrophages via the suppression of MAPKs activation. J Funct Foods 43(4):62-69

31. Jaya SV, Surendra NB, Ruckmani K (2018) Surfactants and fatty alcohol based novel nanovesicles for resveratrol: process optimization, characterization and evaluation of functional properties in RAW 264.7 macrophage cells. J Mol Liq 261(1):387-396
32. Liang J, Li F, Fang Y (2011) Synthesis, characterization and cytotoxicity studies of chitosan-coated tea polyphenols nanoparticles. Colloids Surf B 82(2):297-301

33. Eom TK, Senevirathne M, Kims K (2012) Synthesis of phenolic acid conjugated chitooligosaccharides and evaluation of their antioxidant activity. Environ toxicol phar 34(2):519-527

34. Zhang L, Gui SQ, Wang J (2020) Oral administration of green tea polyphenols (TP) improves ileal injury and intestinal flora disorder in mice with Salmonella typhimurium infection via resisting inflammation, enhancing antioxidant action and preserving tight junction. J Funct Foods 64(3):1-12

35. Vo TS, Ngo DH, Bach LG (2017) The free radical scavenging and antiinflammatory activities of gallate-chitooligosaccharides in human lung epithelial A549 cells. Process Biochem 54(1):188-194

36. Long XY, Pan YN, Zhao X (2018) Prophylactic effect of Kudingcha polyphenols on oxazolone induced colitis through its antioxidant capacities. Food Sci Hum Wellness 7(3):209-214

37. Mei YX, Chen HX, Zhang J (2013) Protective effect of chitooligosaccharides against cyclophosphamide-induced immunosuppression in mice. Int J Biol Macromol 62(11):330-335

38. Nourhan HA, Yasser G, Mohamed ER (2020) Alginate-coated chitosan nanoparticles act as effective adjuvant for hepatitis A vaccine in mice. Int J Biol Macromol 152(3):904-912

\section{Publisher's Note}

Springer Nature remains neutral with regard to jurisdictional claims in published maps and institutional affiliations.

\section{Submit your manuscript to a SpringerOpen ${ }^{\circ}$ journal and benefit from:}

- Convenient online submission

- Rigorous peer review

- Open access: articles freely available online

- High visibility within the field

- Retaining the copyright to your article

Submit your next manuscript at springeropen.com 